\title{
EL CONCEPTO DE SMART DESTINATION EN LA INVESTIGACIÓN TURÍSTICA. REVISIÓN SISTEMÁTICA DE LA LITERATURA PARA SU DEFINICIÓN Y NORMALIZACIÓN
}

\author{
Ainhoa Carballido* \\ Universidad de Málaga \\ https://orcid.org/0000-0002-1401-744X \\ Antonio Guevara-Plaza* \\ Universidad de Málaga \\ https://orcid.org/0000-0002-2400-0668
}

\section{RESUMEN}

Con el auge del uso de las Tecnologías de la Información y la Comunicación (TIC) y un nuevo escenario en el que las zonas urbanas se superponen a las áreas rurales en términos de volumen poblacional, nuevas necesidades emergen, y con ellas nuevos modelos urbanos y económicos como lo son las Smart Cities, las Smart Destinations, o el Smart Tourism. Este artículo trata de establecer una definición holística de estos términos, profundizando en el modelo de inteligencia aplicado al destino turístico, a través de una revisión literaria que permitirá esclarecer los inicios, la evolución y la situación actual de estos conceptos.

Palabras clave: Ciudad Inteligente; Destino Turístico Inteligente; Turismo Inteligente.

The Smart Destination concept in tourism research. a systematic review of the literature for its definition and normalization

\section{ABSTRACT}

With the use of the Information and Communication Technologies (ICT) boom and a new scenario in which urban areas are beyond rural ones in terms of demographic size, new needs emerged, and with them new urban and economic models, such as Smart Cities,

Fecha de recepción: 31 de marzo de 2020.

Fecha de aceptación: 25 de mayo de 2020.

* Facultad de Turismo. Universidad de Málaga. C/ de León Tolstoi, s/n. 29071 MÁlAGA (España). E-mail: ainhoa.carballido@gmail.com,guevara@uma.es 
Smart Destinations, or Smart Tourism. This paper aims to establish a holistic definition of those concepts, deepening in the smartness model applied to a tourism destination, through a literature review that allows clarifying the beginning, the evolution, and the current situation of those concepts.

Keywords: Smart City; Smart Destination; Smart Tourism.

\section{INTRODUCCIÓN}

Durante el inicio del siglo XXI surgen diversos conceptos y terminologías para definir modelos territoriales nuevos o cambiantes, fruto de la necesidad del ser humano a reubicarse como residente de entornos urbanos, integrando las tecnologías de la información y la comunicación (TIC) como parte de su vida cotidiana.

Estos conceptos, giran en torno al significado de la palabra Smart, o Inteligente, en su aplicación al territorio, no solo con la intención de acercarse al modo en que las TIC impactan el mismo, sino tratando de crear modelos de ciudades o municipios sostenibles, accesibles, inclusivos, y conectados, que aboguen por la transparencia, la participación de los diferentes entes sociales y el desarrollo local, elementos necesarios para la buena gobernanza, uno de los pilares descritos como esenciales en el territorio inteligente.

Desde un inicio, autores y expertos como Nam, Pardo, Buhalis, Cohen o Giffinger entre otros, aportan visiones y definiciones propias a este concepto, debatiendo sobre la Ciudad Inteligente (Smart City), el Destino Turístico Inteligente (Smart Destination) y el Turismo Inteligente (Smart Tourism), referidos a lo largo del artículo utilizando sus términos en inglés, desde diferentes corrientes de pensamiento. Es aquí donde surge la necesidad de comprender y definir de un modo holístico y estandarizado estos conceptos, estableciendo nexos y diferencias entre ellos, además de explorar las dimensiones y actores implicados en estos.

Este estudio busca aportar una normalización de la definición de la Smart Destination analizando el contexto del concepto a través de una revisión literaria sistematizada, que aportará resultados cualitativos y cuantitativos permitiendo comprender la evolución del término, así como sus antecedentes y su aplicación, pretendiendo alcanzar con ello los siguientes objetivos específicos (en adelante OE1, OE2, etc.), y sus respectivas preguntas de investigación (en adelante PI1, PI2, etc.):

- OE1: Establecer puntos en común y diferencias entre los conceptos Smart City y Smart Destination

o PI1: ¿Qué es una Smart City?

o PI2: ¿Qué es una Smart Destination?

o PI3: ¿Qué puntos en común y diferencias presentan las definiciones de Smart City y Smart Destination?

- OE2: Contextualizar la Smart Destination como escenario de aplicación de la práctica del Smart Tourism 
o PI4: ¿Qué es el Smart Tourism?

o PI5: ¿Qué se desarrolla en primer lugar el Destino Turístico Inteligente o la práctica del Smart Tourism?

- OE3: Identificar los diferentes modelos de Smart Destination existentes en la actualidad y establecer vínculos entre ellos

o PI6: ¿Qué dimensiones componen los modelos de Smart City y de Smart Destination?

La relevancia de este estudio reside en la unificación de conceptos, la estandarización y normativización del significado del desarrollo de una Smart Destination. Desde un punto de vista teórico, la investigación llevada a cabo pretende generar discusión sobre el conocimiento existente relativo a las Smart Cities, el Smart Tourism y las Smart Destinations, así como en el propio ámbito del Turismo. Entre otras cosas, porque a lo largo de esta investigación se enfrentarán teorías y corrientes de pensamiento alrededor del tema de estudio, algo que comporta, en cierto modo, una epistemología del conocimiento existente.

\section{REVISIÓN LITERARIA: CONTEXTUALIZACIÓN}

Actualmente, más de la mitad de la población mundial (el 55\%) vive en áreas urbanas. Esta cifra ha ido en aumento desde la mitad del siglo XX hasta la actualidad y, según el último informe emitido por Naciones Unidas, se espera que el porcentaje se incremente hasta un $68 \%$ en el año 2050. El crecimiento de la población mundial desde el año 1950 hasta el 2018 (de más de 2,5 millones a más de 7,5 millones) se distribuye de forma desigual en áreas urbanas y rurales, siendo las primeras las más pobladas, especialmente en regiones como Norte América (con un $82 \%$ de su población viviendo en áreas urbanas), Latino América y el Caribe (81\%), Europa (74\%) y Oceanía (68\%). (World Population Prospects The 2017 Revision, 2017)

En este contexto, el territorio urbano se encuentra frente a una nueva realidad que influye en el desarrollo de su propia estructura y requiere de una revisión de sus pilares fundamentales para fomentar el equilibrio y bienestar de su población. Así, algunos territorios urbanos empiezan a autodenominarse Smart Cities con el objetivo de incrementar la calidad de vida de sus habitantes y fomentar la eficiencia de los servicios proveídos tanto por las entidades públicas como privadas (Rodríguez-Bolívar, 2015a), entendiendo el término Smart en su aplicación al territorio como un avance o progreso de un modelo tradicional hacia un modelo digitalizado, o que se adapta a un entorno digital, como ya ocurre en otras áreas más relacionadas con la tecnología aplicada a la comunicación, campo en el que surgió el uso del término como tal, con el nacimiento del primer Smartphone.

Aún así, la comprensión del concepto de inteligencia y su aplicación al territorio o a la actividad turística, variará en función del campo desde el cual se estudie. Los autores Taewoo Nam y Theresa Pardo lo argumentan a través de cuatro visiones diferentes: la del marketing que asocia el término Smart al modo en que el usuario interactúa con el territorio, la de la tecnología que se basa en la aplicación como una extensión del contexto personal hacia la comunidad, y de esta hacia la ciudad, la visión del urbanismo que se centra en la gobernanza mediante el fomento de la relación entre la comunidad y el terri- 
torio, y por último el argumento de las entidades reguladoras que conciben la inteligencia como una oportunidad para crear y distinguir nuevas políticas, estrategias, y planes de acción para el desarrollo sostenible, el crecimiento económico, y la mejora de la calidad de vida de los ciudadanos. (Nam y Pardo, 2011a)

\subsection{Smart City}

No existe una definición única que explique el concepto de Smart City (Nam y Pardo, 2011b), sino diferentes visiones y corrientes de pensamiento, provenientes de distintos autores, de diversos países, en diferentes momentos de las dos últimas décadas. Así, este estudio se centra en presentar dichas definiciones, concretamente aquellas del período comprendido entre los años 2000 y 2018, en las que apreciar como el concepto de Ciudad Inteligente ha ido evolucionando y generando un ecosistema propio.

Las primeras aproximaciones a la descripción del concepto aportan una visión completa y estructurada de lo que se conoce en la actualidad como Ciudad Inteligente, presentando también modelos de dimensiones, actores y factores implicados en el mismo.

En el año 2007, R. Giffinger et al., publicaba el "Smart cities - Ranking of European medium-sized cities" en el que comparaba diferentes ciudades europeas consideradas inteligentes ordenándolas en base a diferentes variables relacionadas con la inteligencia aplicada al territorio. Se basaba en otros rankings anteriores como el publicado en 2003 por Cicille Rozenblat, "Les villes Européennes. Analyse comparative", o el publicado por la Universidad de Mannheim en 2005, “Europas attraktivstes Metropolen für Manger".En esta misma publicación, Giffinger describía la Smart City como una ciudad desarrollada con una visión a largo plazo en todas sus dimensiones, asentada sobre la combinación de diferentes factores que fomenten la autodeterminación, la independencia y la consciencia de sus ciudadanos dotándolos de actividades y recursos específicos.

Tabla 1

DIMENSIONES Y FACTORES DE UNA SMART CITY

\begin{tabular}{|c|c|c|}
\hline $\begin{array}{c}\text { SMART } \\
\text { ECONOMY(competitividad) }\end{array}$ & $\begin{array}{l}\text { SMART GOVERNANCE } \\
\text { (participación ciudadana) }\end{array}$ & $\begin{array}{l}\text { SMART ENVIRO } \\
\text { (medio ambiente) }\end{array}$ \\
\hline $\begin{array}{ll}\text { - } & \text { Innovación y emprendeduría } \\
\text { - } & \text { Imagen económica y marcas } \\
\text { registradas } \\
\text { - } & \text { Productividad } \\
\text { - } & \text { Flexibilidad del mercado } \\
& \text { laboral } \\
\text { - } & \text { Globalización } \\
\text { - } & \text { Capacidad de transformación }\end{array}$ & 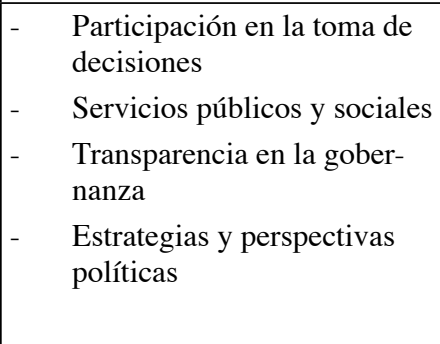 & $\begin{array}{ll}- & \begin{array}{l}\text { Atractivo del entorno } \\
\text { natural }\end{array} \\
\text { - } & \text { Polución } \\
\text { - } & \text { Protección ambiental } \\
\text { - } & \text { Gestión sostenible de } \\
\text { los recursos }\end{array}$ \\
\hline
\end{tabular}




\begin{tabular}{|c|c|c|}
\hline $\begin{array}{c}\text { SMART PEOPLE } \\
\text { (capital social y humano) }\end{array}$ & $\begin{array}{c}\text { SMART MOBILITY } \\
\text { (transportes y accesibilidad) }\end{array}$ & $\begin{array}{l}\text { SMART LIVING } \\
\text { (calidad de vida) }\end{array}$ \\
\hline \begin{tabular}{|ll}
- & Nivel de cualificación \\
- & Aprendizaje continuo \\
- & Pluralidad social y étnica \\
- & Creatividad \\
- & Cosmopolitismo (principio de \\
mente abierta) \\
- & $\begin{array}{l}\text { Participación en la vida pú- } \\
\text { blica }\end{array}$ \\
\end{tabular} & \begin{tabular}{|l} 
Accesibilidad local, nacional \\
e internacional \\
- Infraestructuras tecnológi- \\
cas: existencia y capacidad \\
de uso \\
Sistema de transporte soste- \\
nible, innovador y seguro
\end{tabular} & \begin{tabular}{|ll}
- & Facilidades culturales \\
- & Sanidad \\
- & Seguridad individual \\
- & Calidad de vivienda \\
- & Facilidades educa- \\
& tivas \\
- & Atractivo turístico \\
- & Cohesión social \\
\end{tabular} \\
\hline
\end{tabular}

Fuente: Creación propia a partir de Giffinger et al. (2007)

En estas primeras definiciones, la tecnología forma parte del ecosistema de la Ciudad Inteligente como un componente esencial para la movilidad (el transporte) y el crecimiento de la ciudad en si misma, focalizando el desarrollo de esta en el ciudadano y en su autonomía, garantizando la calidad de vida de estos, y priorizando su integración en la comunidad, haciéndoles sentir parte de la ciudad, parte del proceso de toma de decisión de la misma, y de su desarrollo.

No es hasta dos años más tarde cuando las TIC empiezan a cobrar más protagonismo en la definición de las Smart Cities, cuando se publicaba Helping CIOs Understand "Smart City" Initiatives, artículo en el que se hablaba de la utilización de la tecnología Smart Computing como vehículo para la mejora de los componentes, los servicios, y las infraestructuras de una ciudad, con el objetivo de favorecer su interconexión y eficiencia (Washburn y Sindhu, 2009).

Partiendo de esa base, en el año 2010, los autores Harrison, Eckman, Hamilton, Hartswick, Kalagnanam, Paraszczak, y Williams, afirman que la Smart City debe definirse como una ciudad interconectada, instrumentada e inteligente (Harrison et al., 2010). En esta definición, las Tecnologías de la Información y la Comunicación adquieren un papel importante en su uso aplicado al territorio. En este sentido, los autores explican la instrumentación como el acto de capturar e integrar los datos provenientes de la vida de los habitantes de una ciudad a través de diferentes dispositivos instalados en la misma, como por ejemplo el uso de sensores de medición instalados en diversas infraestructuras de la urbe. Esa misma integración, da pie a la interconexión que definen a través del uso de datos provenientes de empresas privadas, organismos públicos y comunidades que facilitarán el desarrollo de los diferentes servicios de la ciudad y el aumento de la eficiencia de los mismos. En cuanto al pilar de la inteligencia, los autores hablan de la inclusión de analíticas, métricas, optimización y visualización de procesos operacionales que faciliten el proceso de toma de decisiones en el día a día de una ciudad, desde la estructuración de infraestructuras, hasta la organización del sistema de transporte y movilidad de esta.

Un año más tarde, diversos autores apuestan por la inversión en tecnología y el desarrollo de infraestructuras que fomenten la comunicación, así como la integración del capital humano y social, como pilar sobre el que se asienta Wla Smart City. Esta premisa se aborda como palanca para el crecimiento sostenible de la economía, y la gestión de 
los recursos del territorio, todo a través de la participación activa de su comunidad y su gobierno (Caragliu, del Bo, y Nijkamp, 2011). Ese mismo año, los autores Taewoo Nam y Theresa A. Pardo recopilaron y organizaron los orígenes de la Smart City, a través del conocimiento transferido en este ámbito, en base a tres dimensiones: la tecnológica, la social y la comunitaria. Estas tres dimensiones servían para poner de manifiesto el modelo multidimensional de una Ciudad Inteligente, pero también daban una aproximación a la definición de la misma desde tres campos diferentes en los que diversos autores definieron el territorio en base a necesidades y realidades específicas (Nam y Pardo, 2011b).

Continuando con la evolución del concepto de Smart City y, las dimensiones que la componen, en el año 2012, el estratega urbano y climático Boyd Cohen, se basa de nuevo en el modelo de 6 dimensiones de Rudolf Giffinger publicando una adaptación del mismo, con el nombre de Smart City Wheel, un sistema integrado de dimensiones e indicadores que aporta una visión de conjunto sobre los actores implicados en la gestión territorial de una ciudad y los pilares esenciales para que esta sea considerada Smart.

Figura 1

SMART CITY WHEEL

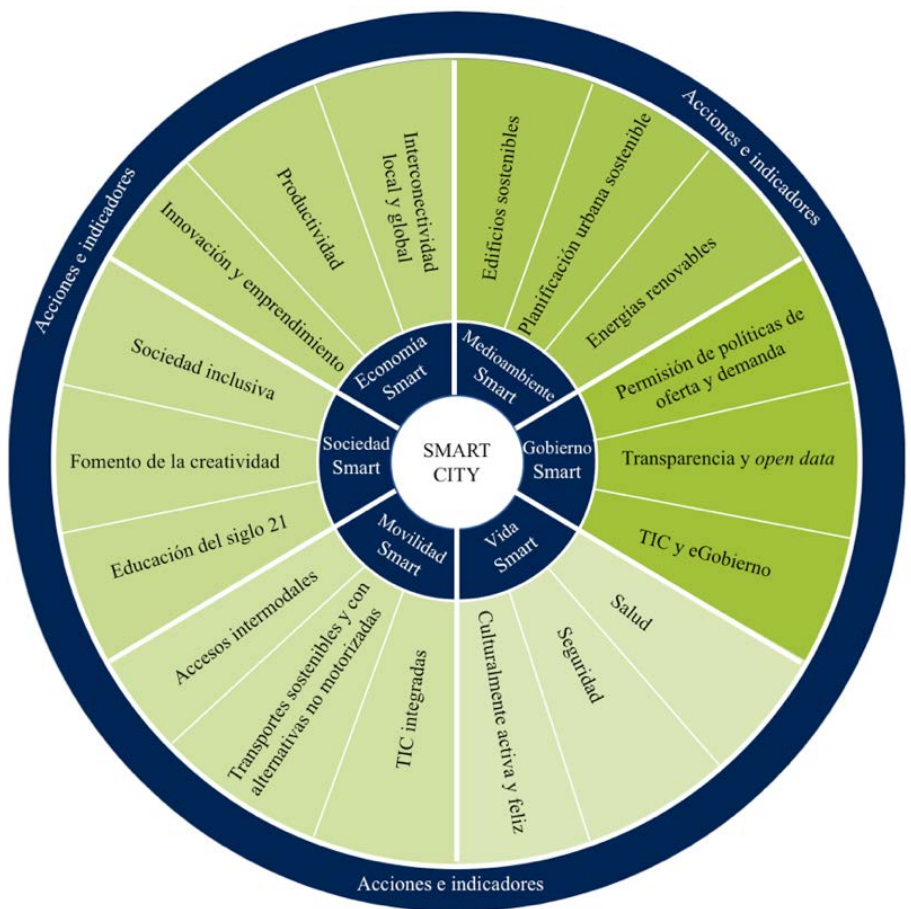

Fuente: Elaboración propia a partir de Boyd Cohen (2012).

Durante los siguientes años, los autores, se centran en la participación ciudadana como eje de la transformación hacia una Smart City, focalizando su atención en el incremento de 
la calidad de vida de sus ciudadanos, mejorando la eficiencia y aumentando la calidad de los servicios que las entidades públicas y privadas proveen a su comunidad (RodríguezBolívar, 2015b).

Basándose en el incremento de la calidad de vida de los ciudadanos (Boes, Buhalis, y Inversini, 2015) como foco principal de la Smart City, en el año 2015 se propone un modelo en el que la gobernanza junto con la innovación y la tecnología (TIC), actúan como columna vertebral del territorio inteligente, formando parte y ejerciendo sobre cinco ámbitos interrelacionados: economía, calidad de vida, medio ambiente, movilidad y ciudadanía, tal como se ejemplifica en la figura 2, en la que se observan dos círculos concéntricos que conectan cada una de las dimensiones representadas con diferentes colores para su mejor comprensión. Así, según las investigaciones llevadas a cabo por el Instituto Valenciano de Tecnologías Turísticas, la gestión de estos ámbitos desde un "punto de vista inteligente" es posible gracias a la inclusión de una estrategia de conjunto pensada y desarrollada específicamente para la Smart City (Invat-tur, 2015).

\section{Figura 2 \\ MODELO DE REFERENCIA DE UNA SMART CITY}

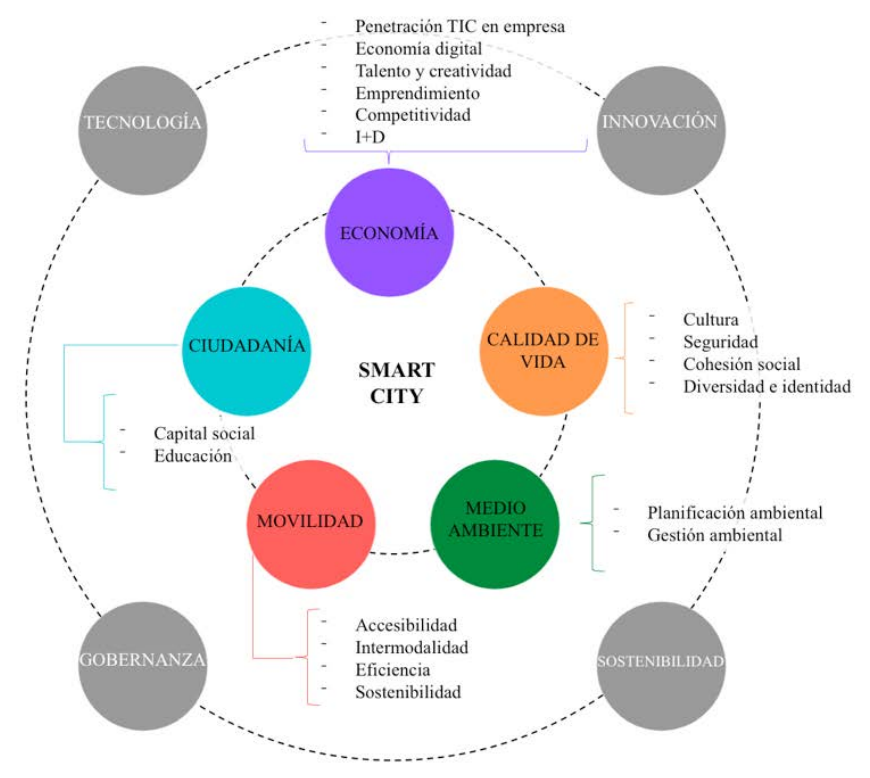

Fuente: Elaboración propia a partir de (Dirks et al., 2009; Giffinger et al., 2007; Telefónica, 2011; Invat·tur, 2015)

\subsection{Smart Tourism}

Partiendo de la actual definición de turismo propuesta por la Organización Mundial del Turismo (2015): 
"Fenómeno social, cultural y económico que implica el movimiento de personas a regiones o lugares diferentes de su entorno habitual de residencia ya sea por una motivación personal o profesional" (UNTWO, 2015).

El Smart Tourism debe ser comprendido como una progresión o avance del turismo tradicional (Xiang, Tussyadiah, y Buhalis, 2015) hacia un nuevo fenómeno social que nace de la convergencia de las Tecnologías de la Información y la Comunicación con el turismo de experiencia. (Hunter, Chung, Gretzel, y Koo, 2015)

En este contexto, el Turismo Inteligente tiene lugar en destinos que cuentan con una infraestructura tecnológica capaz de garantizar el desarrollo sostenible de las diferentes áreas turísticas, fomentando la accesibilidad para todo tipo de personas, y facilitando la integración e interacción de los visitantes con la población local, sirviendo como pilar esencial para el aumento de la calidad de la experiencia turística en el destino y la mejora de la calidad de vida de los propios residentes. (Xiang et al., 2015)

Según los autores Xiang, Tussyadiah, y Buhalis, el Turismo Inteligente se compone de tres pilares: la experiencia, el tejido empresarial, y el territorio. A su vez, estos tres pilares se nutren de los datos que, gracias a la interconexión y a la integración de todos sus componentes y dimensiones, son recogidos, intercambiados, analizados y tratados para facilitar y fomentar el proceso de inteligencia del modelo Smart Tourism (Xiang et al., 2015). Así, la tecnología, la toma de consciencia del entorno por parte de los visitantes, y la monitorización en tiempo real, se convierten en elementos esenciales para la mejora de la experiencia turística a través de la personalización.

Pero para que todo ello sea posible, se advierte imprescindible la interconexión dinamizada de los diferentes stakeholders implicados en la digitalización, tanto en términos organizacionales como operacionales, en aquellos negocios relacionados con la industria turística.

Cabe señalar que el concepto Smart Tourism ha pasado por una evolución significativa en los últimos años y, además, con ciertas diferencias entre las distintas regiones del planeta. Según el artículo Smart Tourism. Un estudio de mapeo sistemático, en Asia del Este su aplicación se centra en la implementación de soluciones tecnológicas, en Oceanía sin embargo, concretamente en Australia, el discurso se inclina más hacia la mejora de los procesos de gobernanza a través de sistemas de información abiertos (open data). Por último, en el caso de Europa "las iniciativas se asocian más a la innovación y competitividad, mediante el desarrollo de aplicaciones para la mejora de la experiencia turística", profundizando de igual modo en el desarrollo sostenible en todas sus escalas o dimensiones ${ }^{1}$ (Celdrán-Bernabéu, Mazón, Ivars-Baidal, y Vera-Rebollo, 2018).

\subsection{Smart Destination}

No es hasta principios de la segunda década de los años 2000 cuando se procede a aplicar el modelo Smart al destino turístico, argumentando que un territorio inteligente

1 Desarrollo sostenible: tras la publicación del Informe Bruntland o Our Future in Common (Brundtland, 1987), y la Agenda 21, esta última como resultado de la Cumbre de la Tierra en 1992, los autores dividen la Sostenibilidad en tres dimensiones: económica, social, y ecológica o medio ambiental (Artaraz Miñón, 2001)1987. Sin embargo, en 2015, Naciones Unidas publicaba lo que supondría la evolución del modelo de tres dimensiones hacia un espectro más global y específico: los 17 Objetivos de Desarrollo Sostenible (Diaz Barrado, 2016). 
se compone de diferentes dimensiones e industrias, entre ellas la turística (Guo, Liu, y Chai, 2014), poniendo de manifiesto la intención de conseguir nuevos modelos de turismo inteligente, y estableciendo así una conexión directa entre los conceptos de Smart City y Smart Destination. De hecho, tal como afirman (Luque, Zayas, y Caro, 2015)“los destinos turísticos inteligentes suponen la evolución natural de las Smart Cities" con elementos y objetivos comunes como la mejora de la gobernanza en el territorio o el aumento de la calidad de vida de sus residentes, añadiendo el incremento de la calidad de la experiencia turística de los visitantes en el caso de la Smart Destination.

Este nuevo contexto, para la terminología de Smart Destination, fue incluido en el Plan Nacional e Integral de Turismo en el Estado Español (PNIT 2012 - 2015), en él se plantea la necesidad de aumentar la calidad y la competitividad de los destinos turísticos fomentando la innovación, la gestión de la experiencia y la integración de las TIC, como elementos clave que aporten valor añadido al mercado turístico. Además de contemplar la tecnificación de los destinos, bajo el concepto de Destinos Inteligentes, alineándose con la tendencia de las Ciudades Inteligentes, siguiendo un mismo patrón de desarrollo, en este caso marcado por la recepción y gestión de los visitantes de un modo sostenible.

Pero, el ecosistema cambiante de la industria turística y la inclusión en la vida cotidiana de las TIC, así como los cambios en las dinámicas del mercado y la evolución de los actores implicados en el propio destino, planteó un nuevo paradigma en el territorio, sensible especialmente para la industria turística. Este nuevo escenario generó una mayor necesidad de personalización y de adaptación a la demanda por parte de las empresas turísticas, resultando de ello la inclusión de herramientas e infraestructuras que dieran respuesta a estas nuevas necesidades (Buhalis y Costa, 2006) buscando mejorar la calidad de la experiencia del turista a través de la integración de las TIC (Neuhofer, Buhalis, y Ladkin, 2012), en primer lugar como herramientas esenciales en el avance del turismo tradicional hacia el Smart Tourism, fomentando la mejora continua de la experiencia del viajero (Wang, Li, y Li, 2013). De este modo, una Smart Destination pasaba a definirse como un territorio compuesto por la estructura básica de un destino turístico pero con la tecnología como columna vertebral del mismo.

Este avance hacia la Smart Destination debía incluir además componentes relacionados con la competitividad, la sostenibilidad, y la inclusividad (Buhalis y Amaranggana, 2013), esta última estrechamente relacionada con la accesibilidad, no solo a nivel de movilidad y de las propias infraestructuras del destino, como en un inicio se definía, sino también a través del fomento de la integración e inclusión de todas las personas en el ecosistema del destino. En este sentido, la tecnología cobra un rol muy activo en el desarrollo del turismo focalizándose así la Smart Destination en la implementación de las TIC como objetivo principal de su estrategia (Wang et al., 2013) (Guo et al., 2014) (Zhu, Zhang, y Li, 2014) (Guevara Plaza y Rossi Jimenez, 2014).

Esta integración de las TICs debe contar además con una arquitectura software capaz de soportar el desarrollo de un Sistema Integrado de Gestión de Destino, para la creación de una red de información externa e interna del propio destino y su tejido empresarial, que permita su gestión integral, y la planificación de una estrategia turística de conjunto (Aguayo-Maldonado, 2015)cabe destacar que a nivel funcional, las TIC desarrollan pape- 
les decisivos en la gestión del destino como el de promoción, información, planificación, o trazabilidad entre otros (Guevara Plaza y Rossi Jimenez, 2014).

Sin embargo, la integración de las tecnologías de la información y la comunicación en el destino no sería suficiente para el desarrollo de una Smart Destination, siendo necesarios otros factores como el capital humano, el liderazgo competitivo, el capital social y la innovación (Boes et al., 2015).Siguiendo esta misma corriente, el Instituto Valenciano de Tecnologías Turísticas (Invat·tur) en colaboración con el Instituto Universitario de Investigaciones Turísticas (IUIT) de la Universidad de Alicante, publicaba en 2015 el Manual Operativo para la configuración de destinos inteligentes, en el que sustentaba que la adaptación de la Smart City a los destinos turísticos debía proceder de un modo escalado y de forma adaptable a cada destino turístico pues no todos tienen las mismas necesidades, estructuras o recursos. Desde este punto de vista, la Smart Destination no se asocia única y exclusivamente a la aplicación de un modelo de Smart City a un destino turístico, sino que se define a través de la implicación de factores como la tecnología, la adaptación de la oferta a la demanda, los cambios en la gestión empresarial, los nuevos modelos de negocio, la eficiencia, la competitividad o la sostenibilidad (Invat-tur, 2015).

En la misma línea, la Sociedad Estatal para la Gestión de la Innovación y las Tecnologías Turísticas publicaba el Informe destinos turísticos inteligentes: construyendo el futuro, integrado en el Libro Blanco de Ciudades Inteligentes, como parte integrante de su estrategia, poniendo de manifiesto la importancia de la práctica de la actividad turística en el territorio español y su impacto en el mismo. Es a raíz de esta situación que, según se plantea en el informe, el Ministerio de Industria, Energía y Turismo, decidió apostar por una transformación del turismo español que apuntara hacia un modelo basado en la innovación, la integración de la tecnología, la sostenibilidad y la accesibilidad (SEGITTUR, 2015).

En Abril de 2016 se incorpora al catálogo de AENOR (Asociación Española de Normalización y Certificación) la norma UNE 178501 (revisada en 2018 por la entidad), con el objetivo de certificar como Smart Destination a aquellos destinos que así lo soliciten. En este caso, trabajando en colaboración con SEGITTUR, el destino pasa un proceso de evaluación que le permite establecer una serie de protocolos para la mejora e innovación del territorio hasta ser considerado Smart. La norma, además de marcar una serie de requisitos, también aporta recomendaciones para el cumplimiento de los mismos, entre ellos el relativo a la importancia de ser un destino turístico consolidado, con datos registrados sobre la actividad turística (afluencia, ocupación, etc.) (AENOR, 2016)"title":’UNE 178501 Sistema de gestión de los destinos turísticos inteligentes. Requisitos.","type":"a rticle"\},"uris":["http://www.mendeley.com/documents/?uuid=741fb248-a5ce-3de8-b3111f36f21b5b20"]\}],"mendeley":\{“formattedCitation":"(AENOR, 2016. Esta norma también establece los ejes sobre los que debe asentarse el destino: innovación, tecnología, accesibilidad universal, y sostenibilidad; orientados estos a la propia prestación turística y no a la gestión del territorio, tal como indican Flores, Perogil, y Miedes, la UNE 178501 "tiene una visión eminentemente productiva y comercial, en la que se deduce no existe una visión de desarrollo local participativo y social" (Flores, Perogil, y Miedes, 2017).

Siguiendo esta misma línea, y centrándose en argumentos relacionados con la integración de las TIC en los destinos turísticos como base para el avance hacia la Smart Destination, A. Cerezo publicaba en su tesis doctoral que el mero uso de tecnología no debe considerarse el único factor para el desarrollo de un Destino Turístico Inteligente ya 
que "las tecnologías por si solas carecen de un valor estratégico si no están respaldadas por un factor humano alfabetizado digitalmente", es por ello que un modelo de gestión Smart debe concebirse como aquel que fomente la cooperación entre los diferentes actores turísticos y territoriales para una gestión inteligente del destino (Cerezo, 2016).

Así, en el año 2017, se constata el modelo y definición establecidos en el 2016, fomentando aún más la sostenibilidad y bienestar de las personas, tanto residentes locales como visitantes, utilizando las TICs como herramientas para la mejora de los destinos, y contemplando nuevas incorporaciones tecnológicas, cada vez más presentes en la vida cotidiana del ser humano, como el Internet de las Cosas (Internet of Things), y el Big Data, de nuevo para la mejora de la experiencia del usuario (Shao, Chang, y Morrison, 2017). Y es que, tal como afirman M. J. Perea-Medina, E. Navarro-Jurado, y A.M. LuqueGil "la forma de hacer turismo está cambiando", actualmente las emociones y las propias experiencias en el destino cobran una mayor relevancia, es por ello que el propio territorio y sus agentes locales deben ser parte integrante de la práctica turística sin dejar que la homogeneización o la estandarización de los destinos afecte a la identidad y la cultura del mismo (Perea-Medina, Navarro-Jurado, y Luque-Gil, 2018).

En última instancia, la Sociedad Estatal para la Gestión de la Innovación y las Tecnologías Turísticas (SEGITTUR), definió la Smart Destination como "un espacio innovador, accesible a todos, apoyado en una estructura tecnológica de vanguardia que garantiza el desarrollo sostenible del territorio turístico y facilita la interacción e integración del visitante con el entorno, incrementando la calidad de su experiencia en el destino" (SEGITTUR, 2018).

\section{METODOLOGÍA}

Teniendo en cuenta los objetivos principales de este trabajo, se plantea un análisis de la producción científica por revisión sistematizada (Jesson, Matheson, y Lacey, 2011) como metodología de investigación, considerando el Framework SALSA (Search, AppraisaL, Synthesis, Analysis) (Samnani, Vaska, Ahmed, y Turin, 2017) como marco de trabajo, el cual comprende cuatro componentes básicos para la consecución de la revisión sistemática que, a su vez, actúan como fases de la misma:

Búsqueda, mediante criterios de inclusión y exclusión, en base a palabras clave, y la utilización de operadores booleanos.

- Evaluación, a través de un sistema de filtrado establecido a partir de los criterios de inclusión y exclusión,

- Síntesis y Análisis, comparando los resultados obtenidos mediante una revisión o narración crítica, seguida de una representación de los resultados a través de esquemas o síntesis, así como de tablas o fichas explicativas.

A partir de este punto se selecciona la base de datos a utilizar como herramienta metodológica del estudio, en este caso Scopus ${ }^{2}$ y Web of Science ${ }^{3}$, y se diseña la estrategia para

2 Scopus: base de datos académica e internacional, con amplia cobertura de publicaciones en Ciencias Sociales en las que se encuentra clasificado el campo de estudio de Turismo y territorio

3 Web of Science: base de datos con alto contenido de publicaciones sobre conceptos Smart, y trabajos enmarcados en el ámbito de las Ciencias Sociales, campo de estudio del Turismo y Territorio. 
seis búsquedas diferentes que se ejecutan utilizando los operadores booleanos indicados a continuación, junto con las palabras clave utilizadas en cada búsqueda:

- Búsqueda 1 (SC): "Smart City” OR "Smart Cities" OR "Ciudad Inteligente” OR "Ciudades Inteligentes".

- Búsqueda 2 (SD): "Smart Destination" OR "Smart Destinations" OR "Destino Inteligente" OR "Destinos Inteligentes" OR "Destino Turístico Inteligente" OR "Destinos Turísticos Inteligentes".

- Búsqueda 3 (ST): "Smart Tourism” OR “Smart Tourist” OR “Turismo Inteligente” OR "Turista Inteligente"

- Búsquedas $4(\mathrm{SC}+\mathrm{SD}), 5(\mathrm{SD}+\mathrm{ST})$, y $6(\mathrm{SC}+\mathrm{SD}+\mathrm{ST})$. Para estas tres búsquedas se realiza una combinación de las anteriores, utilizando el operador AND como nexo para obtener resultados combinados de ambos conceptos.

Una vez ejecutadas las búsquedas, se realiza un filtrado de resultados por fases aplicando los criterios de inclusión y de exclusión para cada una de las seis situaciones de búsqueda.

Los criterios de inclusión y de exclusión utilizados son pragmáticos (lengua de escritura, fecha de publicación, etc.), y de calidad (ámbito geográfico, tema de estudio, metodología utilizada, etc.) tal como se observa en la tabla 2, permitiendo así la selección de una muestra de 103 documentos.

Por último, da comienzo la revisión de los contenidos, a partir de la cual se analizan y sintetizan los resultados a fin de extraer conclusiones plausibles que respondan a los objetivos de la investigación y den respuesta tanto a los resultados cuantitativos esperados como a los cualitativos, apoyándose también el estudio en la realización de un análisis estadístico de textos mediante el uso del software Atlas.ti.

\section{Tabla 2 \\ CRITERIOS DE INCLUSIÓN Y EXCLUSIÓN PARA EL ANÁLISIS DE LA PRODUCCIÓN CIENTÍFICA POR REVISIÓN SISTEMATIZADA}

\begin{tabular}{|c|c|c|c|c|c|c|c|}
\hline \multicolumn{2}{|c|}{ Búsquedas } & $\mathrm{SC}$ & $\mathrm{SD}$ & ST & $\mathrm{SC}+\mathrm{SD}$ & $\mathrm{SD}+\mathrm{ST}$ & $\mathrm{SC}+\mathrm{SD}+\mathrm{ST}$ \\
\hline \multirow{7}{*}{ 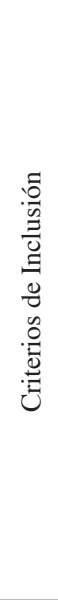 } & 1 & \multicolumn{6}{|c|}{$\begin{array}{l}\text { Trabajos que contengan en su título, resumen o palabras clave los términos de búsqueda, o su } \\
\text { traducción al Español, así como sus variables en plural. }\end{array}$} \\
\hline & 2 & \multicolumn{6}{|c|}{ Trabajos publicados entre los años 2000 y 2018.} \\
\hline & 3 & \multicolumn{6}{|c|}{$\begin{array}{l}\text { Publicaciones con todos los textos completos disponibles ya sea a través de Scopus, Web of } \\
\text { Science, o la editorial de publicación, utilizando para ello el filtro de Acceso Abierto ("Open } \\
\text { Access") en ambas plataformas. }\end{array}$} \\
\hline & 4 & \multicolumn{6}{|c|}{$\begin{array}{l}\text { Tipología de publicaciones incluidas: artículos científicos, libros, capítulos de libro, o } \\
\text { revisiones literarias. }\end{array}$} \\
\hline & 5 & \multicolumn{6}{|c|}{ Publicaciones realizadas en los idiomas: Inglés y Español. } \\
\hline & 6 & \multicolumn{6}{|c|}{$\begin{array}{l}\text { Publicaciones contempladas en el ámbito de Ciencias Sociales, en el que se establece el } \\
\text { ámbito turístico, y no en otros. }\end{array}$} \\
\hline & 7 & \multicolumn{6}{|c|}{$\begin{array}{l}\text { Trabajos que se centren en alguno de los siguientes temas: Definiciones del concepto de } \\
\text { búsqueda, Descripción de modelos de Smart City y/o Smart Destination, Evolución histórica } \\
\text { del concepto de búsqueda, y en el caso del Smart Tourism, descripción de los elementos y } \\
\text { actores presentes en el mismo. }\end{array}$} \\
\hline
\end{tabular}




\begin{tabular}{|c|c|c|c|c|c|c|c|}
\hline \multicolumn{2}{|c|}{ Búsquedas } & $\mathrm{SC}$ & SD & ST & $\mathrm{SC}+\mathrm{SD}$ & $\mathrm{SD}+\mathrm{ST}$ & $\mathrm{SC}+\mathrm{SD}+\mathrm{ST}$ \\
\hline \multirow{6}{*}{ 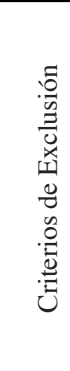 } & 1 & \multicolumn{6}{|c|}{$\begin{array}{l}\text { Que no incluyan en su título, resumen o palabras clave los términos, y sus variantes, } \\
\text { especificados en el criterio de inclusión número } 1 \text {. }\end{array}$} \\
\hline & 2 & \multicolumn{6}{|c|}{ Trabajos publicados antes del año 2000 y/o después del año 2018.} \\
\hline & 3 & \multicolumn{6}{|c|}{$\begin{array}{l}\text { Trabajos sin disponibilidad de texto completo en la plataforma Scopus, Web Of Science, o su } \\
\text { propia editorial. }\end{array}$} \\
\hline & 4 & \multicolumn{6}{|c|}{$\begin{array}{l}\text { Publicaciones que no sean artículos científicos, libros, capítulos de libro, o revisiones } \\
\text { literarias. }\end{array}$} \\
\hline & 5 & \multicolumn{6}{|c|}{ Trabajos publicados en otros idiomas diferentes del Inglés y el Español. } \\
\hline & 6 & \multicolumn{6}{|c|}{$\begin{array}{l}\text { Que su investigación no se centre en ninguno de los temas especificados en el criterio de } \\
\text { inclusión número } 6 .\end{array}$} \\
\hline
\end{tabular}

Fuente: Elaboración propia.

\section{ANÁLISIS Y DISCUSIÓN DE RESULTADOS}

La estrategia de búsqueda diseñada para su implementación en las bases de datos Scopus y Web of Science, se ejecutó la primera semana de Agosto de 2019, devolviendo un total de 19.123 resultados para la primera búsqueda llevada a cabo con la palabra clave Smart City que, tras la aplicación de los diferentes criterios de inclusión y de exclusión deja un total de 544 documentos para su análisis y categorización.

En la tabla 2, se presenta el volumen de producción científica asociado a cada búsqueda. Destacando las 3 búsquedas relativas a los conceptos de Smart City, Smart Destination, y Smart Tourism, las que finalmente reportaron documentos. Se observa una diferencia representativa en el volumen de producción científica cuando se procede a aplicar el criterio de inclusión (CI) y de exclusión (CE) número 3 (libre acceso a los documentos). De igual modo ocurre cuando se aplica el CI 6 (campo de estudio de la publicación: ciencias sociales), evidenciando que las publicaciones relacionadas con los conceptos de estudio asociados al término Smart, acumulan un mayor número de producción científica en otras áreas de estudio tales como las contempladas en el campo de la tecnología.

Tras aplicar el CI 7 (clasificación de los resultados) llevado a cabo mediante un análisis estadístico de textos y su posterior codificación, el resultado es más ajustado y focalizado en la producción científica existente relacionada con la definición de cada uno de los conceptos de estudio, su evolución en el tiempo, o las diferencias entre ellos, así como con el diseño y/o análisis de modelos territoriales asociados a la aplicación de un sistema Smart.

Continuando con más datos de carácter cuantitativo, cabe destacar la representación geográfica en cuanto al volumen de producción científica detectado en la franja temporal establecida para el estudio. En la figura 3 se puede apreciar un mapa de calor que muestra el volumen de publicaciones según el área geográfica de la afiliación a la que se atribuye el estudio, es decir, a la universidad o entidad investigadora a la que pertenece el autor principal, siendo Europa el continente con un mayor volumen de publicaciones con un ranking encabezado por Italia (109 publicaciones), España (71), y el Reino Unido (40), seguido de Asia con un mayor porcentaje de investigaciones publicadas relacionadas con el tema de investigación, especialmente llevadas a cabo por países como Corea del Sur (45), China (32), y la India (31). El tercer continente con un mayor índice de producción científica en relación con el estudio de las Smart Cities y Smart Destinations, así como 
del Smart Tourism, es el continente Americano, con una alta representación de trabajos llevados a cabo por diferentes universidades de Estados Unidos, Brasil, México, Canadá, y Colombia. Destacando la baja representatividad del continente Africano, y el de Oceanía.

\section{Tabla 3 \\ VOLUMEN DE PRODUCCIÓN CIENTÍFICA APLICANDO CRITERIOS DE INCLUSIÓN Y DE EXCLUSIÓN}

\begin{tabular}{|c|c|c|c|c|c|c|c|c|c|c|c|c|c|c|c|}
\hline \multirow[b]{2}{*}{ BÚSQUEDA } & \multicolumn{6}{|c|}{ CRITERIOS DE INCLUSIÓN } & \multicolumn{6}{|c|}{ CRITERIOS DE EXCLUSIÓN } & \multicolumn{3}{|c|}{ RESULTADOS } \\
\hline & 12 & 3 & 4 & 5 & 6 & 7 & 1 & 2 & 3 & 4 & 5 & 6 & WoS & Scopus & Total \\
\hline \multirow[t]{7}{*}{ SC } & & & & & & & & & & & & & 5.129 & 13.994 & 19.123 \\
\hline & & & & & & & & & & & & & 5.071 & 13.974 & 19.045 \\
\hline & & & & & & & & & & & & & 1.019 & 1.879 & 2.898 \\
\hline & & & & & & & & & & & & & 717 & 970 & 1.687 \\
\hline & & & & & & & & & & & & & 618 & 943 & 1.561 \\
\hline & & & & & & & & & & & & & 224 & 320 & 544 \\
\hline & & & & & & & & & & & & & & 87 & 87 \\
\hline \multirow[t]{7}{*}{ SD } & & & & & & & & & & & & & 29 & 44 & 73 \\
\hline & & & & & & & & & & & & & 29 & 44 & 73 \\
\hline & & & & & & & & & & & & & 12 & 6 & 18 \\
\hline & & & & & & & & & & & & & 11 & 5 & 16 \\
\hline & & & & & & & & & & & & & 11 & 5 & 16 \\
\hline & & & & & & & & & & & & & 10 & 5 & 15 \\
\hline & & & & & & & & & & & & & & 4 & 4 \\
\hline \multirow[t]{7}{*}{ ST } & & & & & & & & & & & & & 260 & 263 & 523 \\
\hline & & & & & & & & & & & & & 260 & 263 & 523 \\
\hline & & & & & & & & & & & & & 58 & 39 & 97 \\
\hline & & & & & & & & & & & & & 48 & 32 & 80 \\
\hline & & & & & & & & & & & & & 47 & 31 & 78 \\
\hline & & & & & & & & & & & & & 36 & 25 & 61 \\
\hline & & & & & & & & & & & & & & 15 & 15 \\
\hline
\end{tabular}

Fuente: Elaboración propia.

Estos datos relacionados con el volumen de publicaciones científicas por región devuelven también información sobre los autores con más títulos publicados sobre al tema de estudio, estos son: Chiara Garau, Simon Joss, Rodríguez- Bolívar, Chulmo Koo, Rob Kitchin, Rudolf Giffinger, Ulrike Gretzel, Taewoo Nam, y Theresa A. Pardo; con publicaciones como: "Evaluating Urban Quality: Indicators and Assessment Tools for Smart Sustainable Cities" (Garau y Pavan, 2018), "The smart city and its publics: insights from across six UK cities" (Cowley, Joss, y Dayot, 2018), "SMART Destinations: new strategies to manage tourism industry" (Rodríguez-Bolívar et al., 2015), o "Understanding the Contextual Development of Smart City Initiatives: A Pragmatist Methodology" (Tompson, 2017), entre otras.

Siguiendo con datos de carácter cuantitativo, se plantea una evolución del número de publicaciones a lo largo de la franja temporal delimitada para el estudio. Se observa en la siguiente línea temporal (figura 4) el volumen de producción científica devuelto por las 
bases de datos, aplicando el primer CI y CE, relativo a las palabras claves utilizadas en las ecuaciones de búsqueda.

Figura 3

\section{MAPA VOLUMEN DE PRODUCCIÓN CIENTÍFICA}
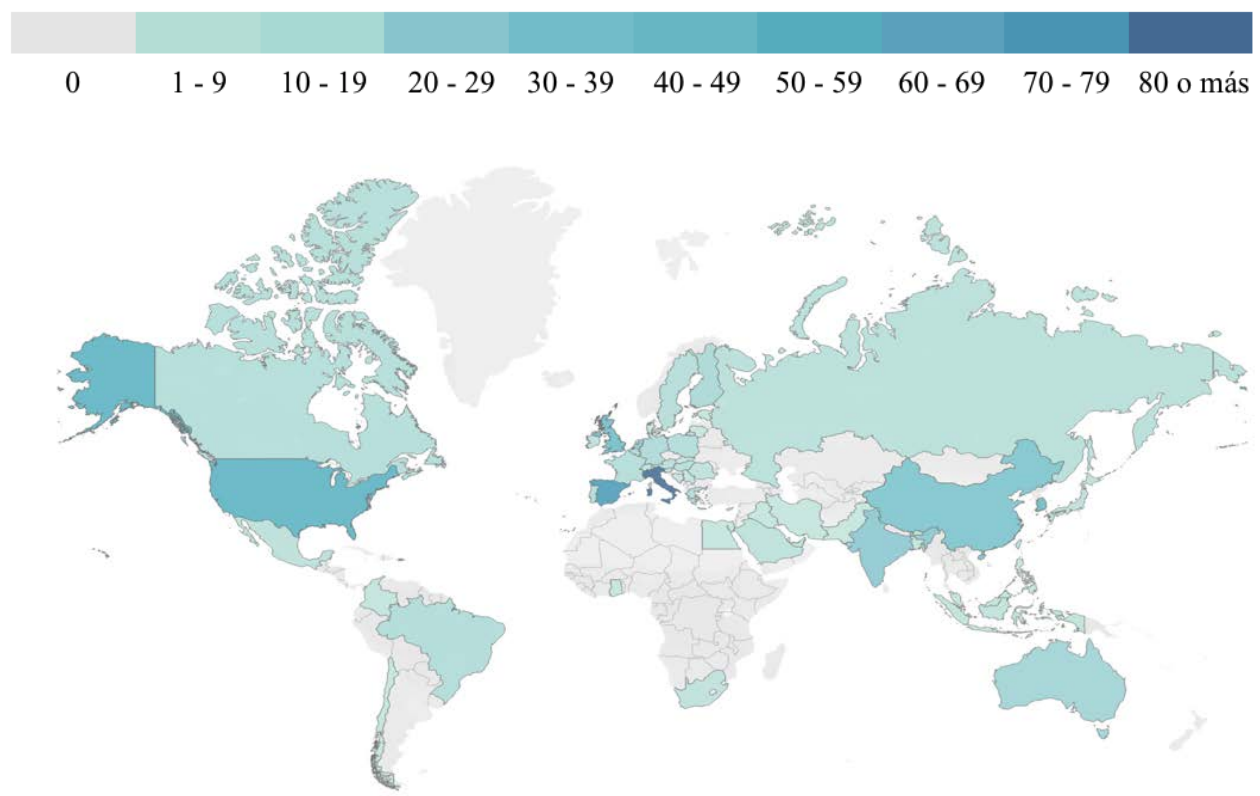

Fuente: Elaboración propia.

Figura 4

\section{LÍNEA TEMPORAL PRODUCCIÓN CIENTÍFICA 00 - 18}

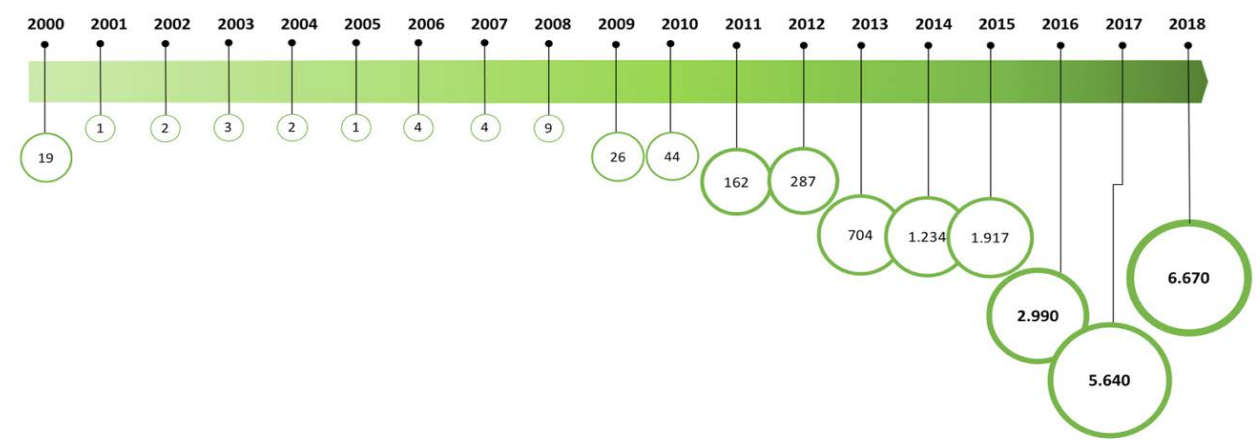

Fuente: Elaboración propia. 
Esta línea permite tener una visión global del trabajo científico sobre el tema de estudio. Advirtiendo que los documentos publicados ascienden de forma relevante en el año 2018, alcanzando un total de 6.640.Situando el punto de inflexión entorno al año 2013 en el que el nivel de producción científica sobre los temas de estudio experimenta un notable crecimiento, coincidiendo con la reciente publicación del Plan Nacional Integral de Turismo 2012-2015, en el que se impulsa el proyecto de Destinos Inteligentes de SEGITTUR, en España. Un año marcado también, por la publicación de la Versión 2.0 del informe Smart cities: ranking of European médium-sized cities, por parte del Centro Regional de Ciencia de la Universidad Tecnológica de Viena, con Rudolf Giffinger como autor principal, impulsado por la Comisión Europea.

Profundizando un poco más en aquellos resultados obtenidos después de las 7 fases de filtrado, se elabora de nuevo una línea temporal de carácter cuantitativo que permite visualizar la evolución del estudio de los conceptos Smart City, Smart Destination y Smart Tourism, de un total de 103 publicaciones. En esta línea temporal, se aprecia un crecimiento del nivel de producción científica comprendido entre los años 2015 y 2018, que se corresponde con la anterior línea temporal global de publicaciones y, denota el creciente interés de la población científica en torno al tema de estudio.

\section{Figura 5 \\ LÍNEA TEMPORAL PRODUCCIÓN CIENTÍFICA 00 - 18 (CI Y CE APLICADOS)}

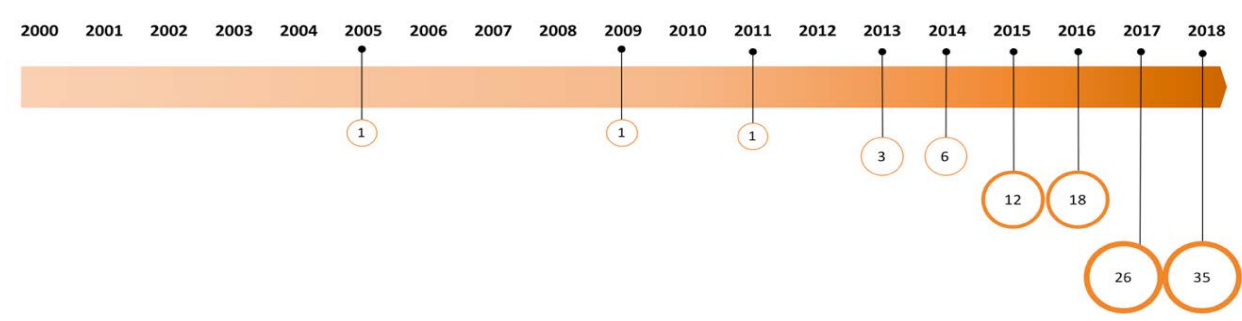

Fuente: Elaboración propia.

Una vez expuestos los resultados cuantitativos relativos a la evolución de los términos, cabe destacar que las principales temáticas halladas en los trabajos publicados son relativas a definiciones, evolución, y análisis comparativos de Smart Destinations y Smart Cities, con un total de 78 publicaciones, seguido de 14 publicaciones relacionadas con modelos y casos de estudio, y finalmente 4 publicaciones sobre la definición del Smart Tourism, y 4 más sobre planes, manuales y/ o guías para la implementación de un sistema Smart en el territorio.

Por último, tras el análisis de los trabajos publicados relacionados con la descripción de modelos y casos de estudio de Smart City/ies y Smart Destination/s, cabe destacar que pese a la evidencia de desarrollo de modelos de gestión de Smart City desde principios del siglo XXI, no se observan modelos de Smart Destination, salvo por el publicado en 
2015 por INVAT·TUR, asentado sobre la gobernanza y basado en la inclusión de las TIC y la innovación como apoyo a la sostenibilidad, a partir de diferentes aplicaciones que permiten ejecutar dicho modelo a escala local, por ejemplo en la recientemente acreditada como Destino Turístico Inteligente según la norma de AENOR, Benidorm.

\section{Figura 6 \\ MODELO DE REFERENCIA DESTINO TURÍSTICO INTELIGENTE}

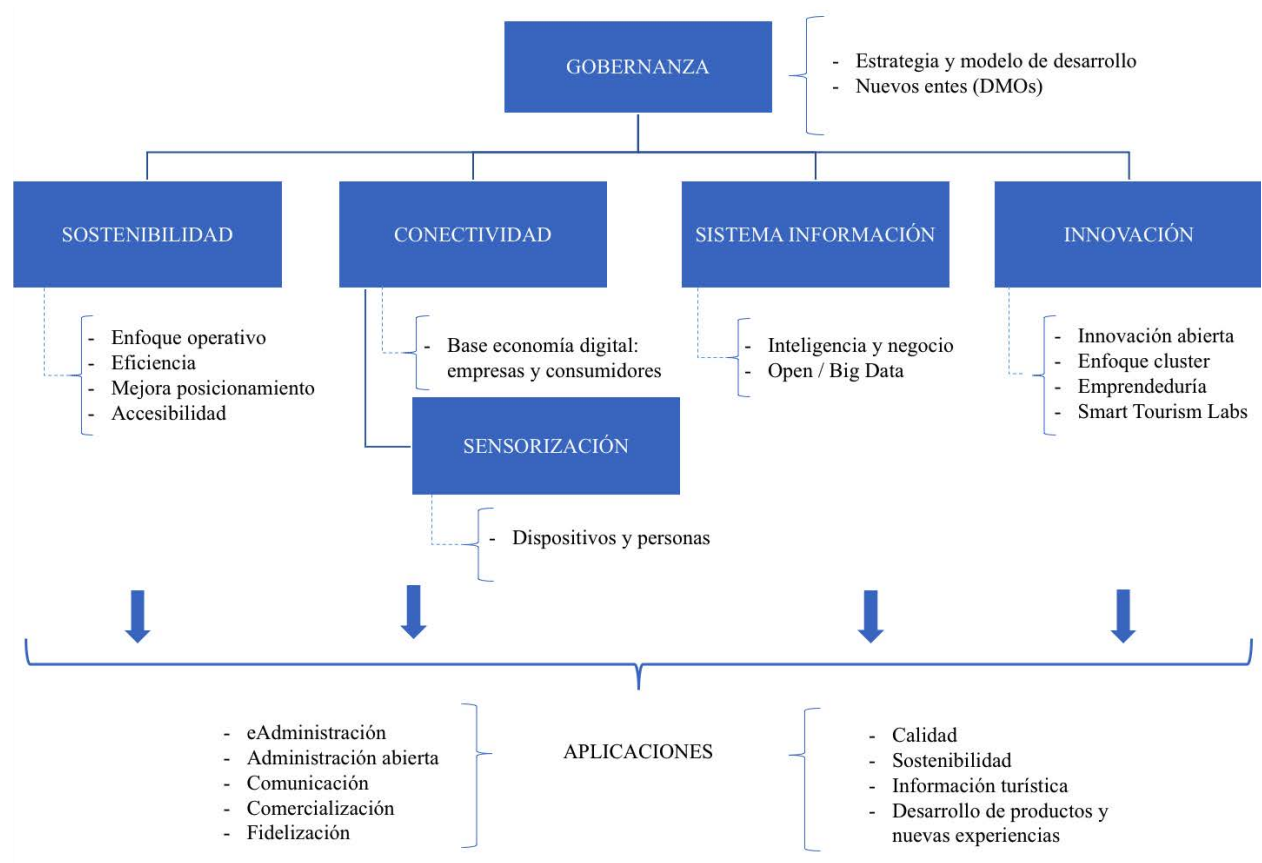

Fuente: Elaboración propia a partir de Invat·tur (2015).

Analizando tanto los modelos de Smart City presentados, así como el modelo de Smart Destination planteado por INVAT·TUR, se propone un nuevo modelo basado en diferentes capas que recoge las dimensiones y los ejes transversales. Este modelo, pretende servir de pauta para la gestión inteligente de un territorio, pertenezca este a una demarcación administrativa (p.ej. la ciudad de Marbella), o a una región supeditada a una marca turística(p. ej. la Costa del Sol a la que pertenece, entre otras ciudades y municipios, la anteriormente citada ciudad de Marbella) para véase en la figura 7.

Este modelo plantea que el sistema se asienta sobre el conjunto de elementos presentes en un territorio, teniendo en cuenta el medio ambiente y los recursos tangibles e intangibles que lo componen. Pero, antes de complementar ese territorio con el tejido empresarial y las entidades públicas que actúan como actores esenciales para la ejecución de la actividad turística, se pone en el centro de la ecuación a la capa social, que incluye a la comunidad local y a la comunidad visitante, como entes principales del sistema Smart, 
abogando por su integración, así como la interacción de ambos grupos sociales. En las capas superiores de este modelo se encuentran los servicios básicos y complementarios, haciendo referencia con ello, a los elementos esenciales para la vida cotidiana, y a otros que permiten mejorar la experiencia del visitante sin degradar la calidad de vida de la comunidad residente.

\section{Figura 7}

\section{PROPUESTA MODELO DE CAPAS PARA LA APLICACIÓN DE UN SISTEMA SMART EN EL TERRITORIO}

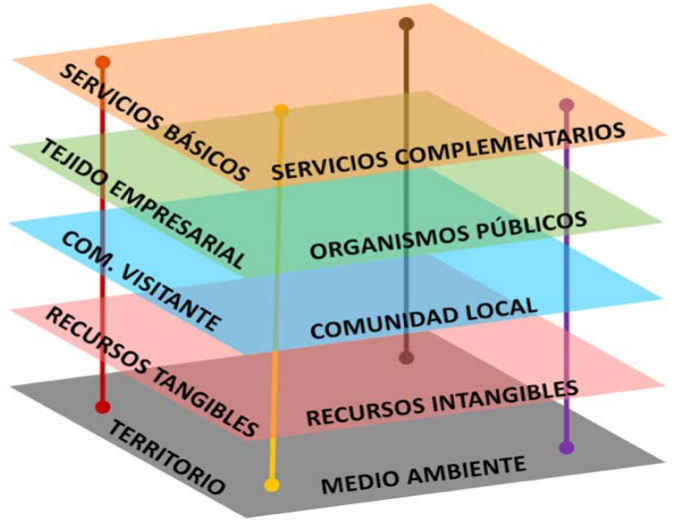

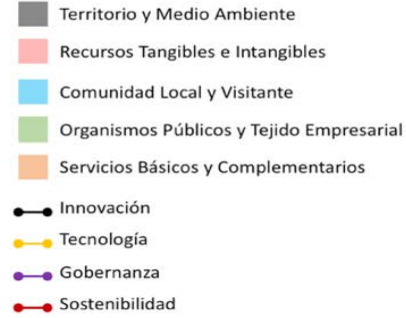

Fuente: Elaboración propia.

Las líneas verticales, representan los ejes principales del modelo de territorio inteligente, ejes que representan los pilares del mismo, sin los cuales sería imposible hablar de una Smart City o una Smart Destination:

- Sostenibilidad: presente en todas y cada una de las capas de un territorio inteligente, contemplando las tres áreas principales de la sostenibilidad (económica, socio-cultural, y medioambiental) como intrínsecas en cada capa, y a su vez, interconectadas entre sí.

- Tecnología: como medio para promover buenas prácticas en el territorio, una mejora de la experiencia tanto de la comunidad visitante como de la local, la preservación del medio ambiente, el fomento de la identidad, y la consecución de un territorio más eficiente, más competitivo, más sostenible.

- Gobernanza: actuando como el elemento regulador, con políticas que aboguen por la sostenibilidad y la innovación, que incorpore la transformación digital en los tejidos públicos y privados, para adecuarse a las nuevas tendencias, y mejorar la accesibilidad e inclusividad de todos los seres humanos, fomentando el respeto y la tolerancia, no solo en lo relativo a la capa social, sino también en la relación de esta con el medio que les rodea. Promoviendo también la participación ciudadana como elemento indispensable de la Smart City o Smart Destination. 
- Innovación: de igual modo que la tecnología, la innovación actúa como un pilar esencial en la búsqueda de buenas prácticas, ensalzando la creatividad, la emprendeduría, y la intraemprendeduría como elementos diferenciadores de una sociedad Smart.

\section{CONCLUSIONES}

A lo largo de este estudio se ha desarrollado un análisis de la producción científica por revisión sistematizada.

En primer lugar, se ejecutaron diferentes búsquedas en dos bases de datos científicas (Scopus y Web of Science) estableciendo diferentes criterios de inclusión y de exclusión, que permitieron un filtrado de los resultados y la obtención de una muestra representativa de las publicaciones halladas entre los años 2000 y 2018. Concretamente se evaluaron un total de 103 trabajos, entre ellos artículos, libros, y/o capítulos de libros, que versaban sobre temáticas relacionadas con las Smart Destinations, las Smart Cities, y/o el Smart Tourism. Y, en una fase de síntesis y análisis, se compararon los resultados obtenidos, tanto cuantitativos como cualitativos, permitiendo obtener datos relevantes en cuanto a la evolución de los conceptos, y la definición de los mismos.

Pese a que en diferentes publicaciones de principios de siglo ya se habla de Smart Cities, y de su relación con temas de índole tecnológica, no es hasta 2007 cuando Rudolf Giffinger propone una definición de Smart City (Giffinger et al., 2007) basada en un modelo de gestión inteligente con 6 dimensiones: economía, gobernanza, medio ambiente, movilidad, social, personas, y vida cotidiana; sin focalizarlo estrictamente en la tecnología, en contraposición a otros autores a lo largo de la evolución del concepto, entre ellos, Theresa Nam y Taewoo Pardo (Nam y Pardo, 2011), quienes en 2011 postulaban que la ciudad debe estructurarse en base a factores tecnológicos, humanos e institucionales que den lugar a un sistema interconectado, proponiendo un modelo segmentado en 3 dimensiones, la humana, la tecnológica y la institucional.

Por tanto, los trabajos publicados en la primera década de este siglo, apuntan a que las Smart Cities nacen con el objetivo de incrementar la calidad de vida de sus habitantes y fomentar la eficiencia de los servicios proveídos tanto por las entidades públicas como privadas, pero se focalizan en el uso de la tecnología como elemento esencial de sus propuestas de modelos de gestión, sin tener en cuenta aspectos relacionados con el turismo, ni integrar definiciones de Smart Destinations o Smart Tourism.

Sin embargo, en los trabajos publicados en la última etapa analizada (2015 - 2018), autores como Hunter, Chung, Gretzel, Koo, Xiang, Tussyadiah, o Buhalis, describen el Smart Tourism como una progresión o avance del turismo tradicional hacia un nuevo fenómeno social que nace de la convergencia de las tecnologías de la información y la comunicación, con el turismo de experiencia, y que tiene lugar en destinos que cuentan con una infraestructura tecnológica capaz de garantizar el desarrollo sostenible de las diferentes áreas turísticas, fomentando la accesibilidad, la integración, y la interacción de los visitantes con la población local, sirviendo como pilar para el aumento de la calidad de la experiencia turística en el destino y la mejora de la calidad de vida de los residentes, este escenario está representado por la Smart Destination. 
Esta evolución en los conceptos apunta hacia el surgimiento de la Smart Destination con un reto latente: la innovación y la gestión de la experiencia, integrado todo ello con el uso de la tecnología, no como objetivo final sino como herramienta para alcanzar un territorio más sostenible, más eficiente y con un mayor valor añadido.

En el análisis de producción científica realizado en este trabajo, se ha conseguido cumplir los objetivos planteados, ya que quedan evidenciadas las diferentes definiciones de los conceptos investigados, los modelos, la evolución de los mismos, así como las respuestas a las preguntas de investigación, las cuales presentamos a modo de conclusión sintetizada en los próximos párrafos.

Una Smart City es aquella que nace con el objetivo de incrementar la calidad de vida de sus habitantes, utilizando la tecnología con el fin de alcanzar un desarrollo sostenible, que abogue por la eficacia de los recursos disponibles, la accesibilidad e inclusividad, la eficiencia, y la autodeterminación. De igual modo, la definición de Smart Destination se refiere a un territorio que promueve el aumento de la calidad y la competitividad de los enclaves turísticos, fomentando la innovación y la gestión de la experiencia del visitante, sin olvidar el bienestar de la comunidad local y la preservación del entorno, todo ello mediante el uso de las TICs. No obstante, pese a que ambos conceptos se definen de un modo similar, en lo relativo a las dimensiones sociales, la Smart Cityse focaliza en la comunidad local y, la Smart Destination, busca un equilibrio entre la comunidad local y la comunidad visitante, fomentando la mejora de la experiencia del turista a través del desarrollo de la economía local, directamente conectada con los residentes del territorio.

En cuanto al concepto de Smart Tourism, basándonos en la propia definición de Smart, el Turismo Inteligente, se describe como una progresión del turismo tradicional hacia una actividad o fenómeno que incluye las TICs, y la experiencia del turista, como base en su práctica. Es por ello que, la ejecución de la actividad turística inteligente no puede tener lugar en un escenario no considerado Smart en sí mismo. Tal como afirma Xiang, entre otros autores (Xiang et al., 2015), y como se menciona anteriormente, el Smart Tourism debe tener lugar en destinos que cuenten con una infraestructura tecnológica capaz de garantizar el desarrollo sostenible, la accesibilidad, la inclusividad, y la interacción visitante-local. Por tanto, podemos afirmar que la Smart Destination es el escenario de la práctica del Smart Tourism, y que esta debe evolucionar o desarrollarse antes de acoger una actividad turística considerada inteligente.

Continuando ahora con los modelos de gestión identificados, en la última etapa de la franja temporal analizada, tanto el modelo de Smart City como el de Smart Destination, contempla cinco dimensiones: economía, ciudadanía, movilidad, calidad de vida, y medio ambiente; además de dos cuatro ejes centrales: tecnología, innovación, gobernanza, y sostenibilidad. Teniendo en consideración este modelo, y todos los aportes de los trabajos analizados, en este estudio se contempla un nuevo modelo de gestión inteligente basado en diferentes capas (territorio y medio ambiente, recursos tangibles e intangibles, comunidad local y visitante, organismos públicos y tejido empresarial, servicios básicos y complementarios) que permite visualizar no solo las diferentes dimensiones y ejes transversales existentes (innovación, tecnología, gobernanza, y sostenibilidad) en un territorio Smart, sino también los actores implicados, factores a tener en cuenta en la búsqueda de indicadores para la medición del elemento Smart. 
Cabe destacar las limitaciones halladas en el desarrollo de este estudio, relacionadas con el bajo volumen de trabajos disponibles con acceso abierto en las dos bases de datos utilizadas, así como la modernidad o frescura de los términos investigados, con apenas una década de historia.

Para concluir, después de realizar esta aproximación al tema de estudio, en búsqueda de una definición holística y del diseño de un modelo de dimensiones de la Smart Destination, la línea futura a la que da pie esta investigación, debe estar relacionada con el análisis de modelos de referencia, así como la descripción de los actores y factores implicados en cada dimensión, para acabar profundizando en el estudio de cada uno de ellos y sus impactos en el desarrollo del sistema Smart en el territorio.

Declaración responsable: Los autores declaran que no existe ningún conflicto de interés en relación con la publicación de este artículo. Las tareas se han distribuido del modo siguiente: el diseño general del artículo ha sido obra de Ainhoa Carballido Risco y Antonio Guevara Plaza, el apartado de metodología y de análisis y discusión de resultados ha sido elaborado por Ainhoa Carballido Risco. La aplicación metodológica, decisiones al respecto, redacción del texto y revisión bibliográfica ha sido responsabilidad de ambos autores, Ainhoa Carballido Risco y Antonio Guevara Plaza.

\section{REFERENCIAS}

ASOCIACIÓN ESPAÑOLA DE NORMALIZACIÓN Y CERTIFICACIÓN (AENOR. (2016): UNE 178501 Sistema de gestión de los destinos turísticos inteligentes. Requisitos. Madrid. Disponible en: https://www.aenor.com/normas-y-libros/buscador-denormas/une?c=N0060239

AGUAYO-MALDONADO, A. (2015): Diseño de un Sistema Integrado de Gestión de Destinos Turísticos. Tesis Doctoral. Universidad de Málaga.

ARTARAZ, M. (2001): «Teoría de las tres dimensiones de desarrollo sostenible», Ecosistemas, vol. X (3), pp. 1-6.

BOES, K., BUHALIS, D. e INVERSINI, A. (2015): «Conceptualising smart tourism destination dimensions», en Information and communication technologies in tourism. Springer, Cham, pp. 391-403.

IMPERATIVES, S. (1987): Report of the World Commission on Environment and Development: Our common future, vol. 14 (4), pp. 291-294.

BUHALIS, D. y AMARANGGANA, A. (2013): «Smart tourism destinations», en Information and communication technologies in tourism 2014. Springer, Cham, pp. 553564.

BUHALIS, D. y COSTA, C. (Eds.) (2006): Tourism management dynamics: trends, management and tools. Routledge.

CARAGLIU, A., DEL BO, C. y NIJKAMP, P. (2011): Smart cities in Europe. Routledge CELDRÁN-BERNABÉU, M.A., MAZÓN, J.N., IVARS-BAIDAL, J.A. y VERAREBOLLO, J.F. (2018): «Smart tourism. Un estudio de mapeo sistemático», Cuadernos de Turismo, $\mathrm{n}^{\circ}$ 41, pp. 107-138. 
CEREZO, A. (2016): Análisis de percepciones y necesidades formativas en tecnologías de la información y la comunicación en turismo. El caso de Andalucía. Tesis Doctoral. Universidad de Málaga.

COWLEY, R., JOSS, S. y DAYOT, Y. (2018): «The smart city and its publics: insights from across six UK cities», Urban Research \& Practice, vol. 11 (1), pp. 53-77.

DIAZ BARRADO, C.M. (2016): "Los objetivos de desarrollo sostenible: un principio de naturaleza incierta y varias dimensiones fragmentadas", Anuario Español de Derecho Internacional, $\mathrm{n}^{\mathrm{o}} 32$, pp. 9-48.

DIRKS, S. y KEELING, M. (2009): «A vision of smarter cities: how cities can lead the way into a prosperous and sustainable future», IBM Institute for business value, $\mathrm{n}^{\circ} 8$.

FLORES, D., PEROGIL, J. y MIEDES, B. (2017): «¿Destinos turísticos inteligentes o territorios inteligentes? Estudios de casos en España», Revista de Estudios Regionales, $\mathrm{n}^{\circ} 113$, pp. 193-219.

FUNDACIÓN TELEFÓNICA. (2011): Smart Cities: un primer paso hacia la internet de las cosas, vol. 16.

GARAU, C. y PAVAN, V.M. (2018): «Evaluating Urban Quality: Indicators and Assessment Tools for Smart Sustainable Cities», Sustainability, vol. 10 (3), p. 575.

GIFFINGER, R., FERTNER, C., KRAMAR, H. y MEIJERS, E., (2007): Smart cities Ranking of European medium-sized cities. Vienna, Centre of Regional Science, Vienna University of Technology.

GUEVARA PLAZA, A. y ROSSI JIMENEZ, C. (2014): «Las TICs aplicadas a la gestión de destinos turísticos», en Manual de gestión de destinos turísticos. Valencia, Tirant lo Blanch, pp. 243-273.

GUO, Y., LIU, H. y CHAI, Y. (2014): «The embedding convergence of smart cities and tourism internet of things in China: An advance perspective», Advances in Hospitality and Tourism Research (AHTR), vol. 2 (1), pp. 54-69.

HARRISON, C., ECKMAN, B., HAMILTON, R., HARTSWICK, P., KALAGNANAM, J., PARASZCZAK, J. y WILLIAMS, P. (2010): «Foundations for Smarter Cities», IBM Journal of Research and Development, vol. 54 (4), pp. 1-16.

HUNTER, W.C., CHUNG, N., GRETZEL, U. y KOO, C. (2015): «Constructivist Research in Smart Tourism», Asia Pacific Journal of Information Systems, vol. 25 (1), pp. 105120.

INVAT·TUR (AGÈNCIA VALENCIANA DEL TURISME) (2015): Destinos turísticos inteligentes: manual operativo para la configuración de Destinos Turísticos Inteligentes. Alicante, Universidad de Alicante. Instituto Universitario de Investigaciones Turísticas.

JESSON, J., MATHESON, L. y LACEY, F.M. (2011): «Doing Your Literature Review :Traditional and Sistematic Techniques. Sage Publication Ltd.

LUQUE GIL, A.M., ZAYAS FERNÁNDEZ, B. y CARO HERRERO, J.L. (2015): «Los Destinos Turísticos Inteligentes en el marco de la Inteligencia Territorial: conflictos y oportunidades», Investigaciones Turísticas, $\mathrm{n}^{\circ} 10$, pp. 1-25.

NAM, T. y PARDO, T.A. (2011): «Conceptualizing smart city with dimensions of technology, people, and institutions», en Proceedings of the 12th Annual International 
Digital Government Research Conference on Digital Government Innovation in Challenging Times. New York, pp. 282-291.

NEUHOFER, B., BUHALIS, D. y LADKIN, A. (2012): «Conceptualising technology enhanced destination experiences», Journal of Destination Marketing and Management, vol. 1 (1-2), pp. 32-46.

PEREA-MEDINA, M.J., NAVARRO-JURADO, E. y LUQUE-GIL, A.M. (2018): «Inteligencia territorial: Conceptualización y avance en el estado de la cuestión. Vínculos posibles con los destinos turísticos», Cuadernos de Turismo, $\mathrm{n}^{\circ}$ 41, pp. 535-554.

RODRÍGUEZ-BOLÍVAR, M.P. (2015): Transforming city governments for successful smart cities. Springer.

RODRÍGUEZ-BOLÍVAR, M.P., YOVANOF, G.S., HAZAPIS, G.N., WASHBURN, D., SINDHU, U., NEUHOFER, B. y MARSAL-LLACUNA, M.L. (2015): «Smart Destinations: new strategies to manage tourism industry», Journal of Destination Marketing and Management, vol. 128 (3), pp. 1.193-1.216.

SAMNANI, S.S., VASKA, M., AHMED, S. y TURIN, T.C. (2017): «Review Typology: The Basic Types of Reviews for Synthesizing Evidence for the Purpose of Knowledge Translation», Journal of the College of Physicians and Surgeons Pakistan, vol. 27 (10), pp. 635-641.

SEGITTUR. (2015): Informe destinos turísticos inteligentes: construyendo el futuro», en Libro Blanco de los Destinos Turísticos Inteligentes. Madrid.

SEGITTUR. (2018): Destinos Turísticos Inteligentes. Extraído el 30 de Julio de 2019 de: https://www.segittur.es/es/DTI/dti-detalle/Destinos-Tursticos-Inteligentes-00007/\#. XUCGMpMzb6Y

SHAO, J., CHANG, X. y MORRISON, A. (2017): «How Can Big Data Support Smart Scenic Area Management? An Analysis of Travel Blogs on Huashan», Sustainability, vol. 9 (12), p. 2.291.

TOMPSON, T. (2017): «Understanding the Contextual Development of Smart City Initiatives: A Pragmatist Methodology», She Ji: The Journal of Design, Economics, and Innovation, vol. 3 (3), pp. 210-228.

UNITED NATIONS (2017): World population prospects: the 2017 revision, key findings and advance tables. New York, Departament of Economics and Social Affairs PD. New York.

WANG, D., LI, X. y LI, Y. (2013): «China's “smart tourism destination” initiative: A taste of the service-dominant logic», Journal of Destination Marketing and Management, vol. 2 (2), pp. 59-61.

WASHBURN, D. y SINDHU, U. (2009): «Helping CIOs Understand "Smart City" Initiatives», Growth, vol. 17 (2), pp. 1-17.

XIANG, Z., TUSSYADIAH, I. y BUHALIS, D. (2015): «Smart destinations: Foundations, analytics, and applications», Journal of Destination Marketing and Management, vol. 4 (3), pp. 143-144.

ZHU, W., ZHANG, L. y LI, N. (2014): «Challenges, Function Changing of Government and Enterprises in Chinese Smart Tourism», Information and Communication Technologies in Tourism, vol. 10, pp. 553-564. 